\title{
Bandpass switching in a nonlinear-optical loop mirror
}

\author{
D. A. Pattison, P. N. Kean, W. Forysiak, I. Bennion, and N. J. Doran \\ Photonics Research Group, Department of Electronic Engineering and Applied Physics, Aston University, Birmingham B4 7ET, UK
}

Received September 13, 1994

\begin{abstract}
We demonstrate bandpass nonlinear switching, using a novel device configuration based on a nonlinear-optical loop mirror and an in-fiber Bragg grating. Self-switching is demonstrated in the soliton regime by use of an asymmetrically arranged in-fiber Bragg grating as a wavelength-selective element. In addition, we adapt the configuration to perform efficient two-wavelength switching.
\end{abstract}

The potential importance of the nonlinear-optical loop mirror (NOLM) in all-optical switching applications is widely recognized. ${ }^{1}$ Ultrafast switching in the NOLM relies on breaking the loop symmetry to achieve a differential phase shift of $\pi \mathrm{rad}$ between counterpropagating pulses in the device. Self-switching is achieved by use of an asymmetric coupler $^{2}$ or by the inclusion of an asymmetrically located gain or loss element. ${ }^{3}$ By using two independent data streams at different wavelengths one can also effect switching through the cross-phasemodulation asymmetry induced by a strong control pulse on a weaker signal pulse. ${ }^{4}$ This technique is now routinely used to perform multigigabit timedivision demultiplexing. ${ }^{5}$

In both regimes of operation the broadband nature of the response limits the applicability of the NOLM in configurations in which the wavelength performance of individual switching elements must be tightly controlled. Consequently the basic NOLM must be modified for use in wavelength-divisionmultiplexed systems in which a narrow bandwidth is essential.

In this Letter we use a novel device configuration to demonstrate wavelength-confined, or bandpass, switching in a NOLM. We demonstrate selfswitching in the soliton regime, using a partially reflecting Bragg grating as a wavelength-dependent loss element, yielding applications in multichannel wavelength-division multiplexing using solitons. ${ }^{6}$ In addition, we demonstrate two-wavelength operation in which a signal is switched by use of crossphase modulation owing to a high-power control signal at a second wavelength that is confined to one arm of the NOLM, using a fiber Bragg grating. In both schemes we observe that operation of the device is confined to wavelengths defined by the grating reflection band.

The potential of the Bragg grating in nonlinear all-optical switching has long been recognized, ${ }^{7}$ but, in practice, switching in the fiber grating has required prohibitively high switching powers. ${ }^{8}$ By combining a grating with a NOLM we retain the wavelength selectivity inherent in the grating but reduce the required switching power by several orders of magnitude.

A schematic of the first experimental configuration is shown in Fig. 1. Transform-limited sech ${ }^{2}$ pulses with a full width at half-maximum of $7 \mathrm{ps}$ from a tunable color-center laser were coupled into the NOLM through the $90 \%$ arm of a 90/10 coupler. The laser was synchronously pumped with a Nd:YAG laser operating at $1064 \mathrm{~nm}$ with a repetition rate of $76 \mathrm{MHz}$. The NOLM was constructed by use of a variable-ratio coupler and $2.4 \mathrm{~km}$ of standard fiber. We implemented the asymmetric loss element, using a chirped in-fiber Bragg grating centered at $1550 \mathrm{~nm}$ with a bandwidth of $1.9 \mathrm{~nm}$ and a peak reflectivity of $60 \%$. The grating was produced in this laboratory by holographic exposure of boron-codoped fiber. ${ }^{9}$ The insertion loss of the configuration was measured to be $6 \mathrm{~dB}$ when the color center laser lay inside the grating reflection profile. The NOLM was operated in linear transmission to isolate the nonlinear response from grating reflections. In this mode the polarization of the nonlinearly reflected signal is orthogonal to that of the input signal and hence also to the portion of the input reflected by the grating. ${ }^{10}$ The nonlinear response was viewed by use of the appropriate port of the polarizing beam splitter. To ensure that any observed nonlinear switching was due solely to the grating, we needed to compensate for asymmetry arising from other sources, e.g., splice loss. We accomplished this by tuning the color-center laser to a wavelength shorter than the grating-reflection peak and varying the coupler splitting ratio until the nonlinear response of the device was extinguished. Under these conditions the grating is transparent to pulses propagating in the loop, and any switching observed is due to other sources of asymmetry. The time-averaged response of the NOLM with the laser tuned to the grating-reflection peak $(\lambda \sim 1550 \mathrm{~nm})$ is shown in Fig. 2(a). The first and second peaks occur at average input powers (soliton numbers) of $3.1 \mathrm{~mW}$ $(N=2.3)$ and $6.8 \mathrm{~mW}(N=3.4)$, respectively.

It is clear from Fig. 2(b), which shows the response of the device when the laser is tuned off the grating peak to $1546 \mathrm{~nm}$, that the switching response is virtually extinguished at wavelengths outside the grating reflection band. The wavelength discrimination of the configuration is therefore $4 \mathrm{~nm}$, a value that is ultimately limited by the bandwidth of the source used and the choice of grating. In this demonstration we used a chirped fiber grating, although a linear grating could equally have been used. Indeed, the substitution of narrow-bandwidth linear gratings would 


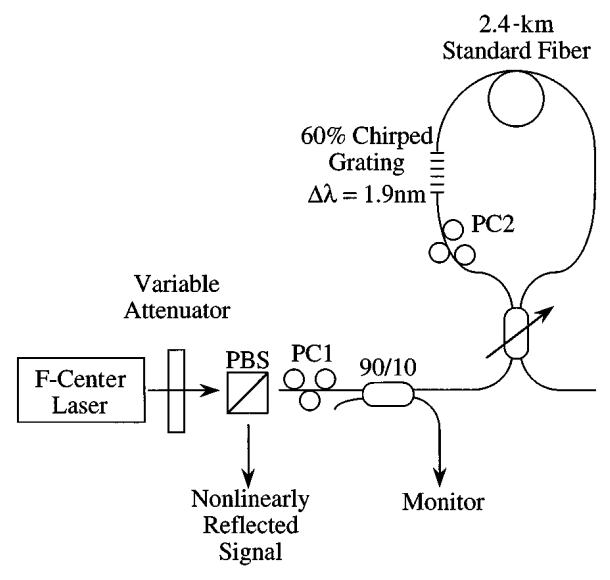

Fig. 1. Schematic of the experimental arrangement to demonstrate wavelength-selective bandpass selfswitching in the soliton regime. The color- $(\mathrm{F}-)$ center laser pulse width is $7 \mathrm{ps}$. PBS, polarizing beam splitter; $\mathrm{PC} 1, \mathrm{PC} 2$, polarization controllers.


Fig. 2. Time-averaged response of the NOLM to the input pulse stream for an incident wavelength lying (a) inside and (b) outside the grating reflection band.

result in enhanced wavelength performance, leading to potential applications in high-density wavelengthdivision multiplexing. Theoretical and experimental study has shown that the quality of pulses transmitted through a chirped grating is degraded only when the reflection band edge is extremely sharp. The apodized band edges of the grating used ensured that the signal pulses emerging from the grating retained their temporal and spectral characteristics, and no degradation of the time-bandwidth product was observed.

By increasing the reflectivity of the grating toward $100 \%$ we see that a signal incident on the NOLM at a wavelength within the grating reflection band will be restricted to only one arm of the device. Us- ing this principle, we use a high-power control pulse of wavelength $\lambda_{1}$, which lies within the grating reflection characteristic, to switch a signal pulse at a second wavelength $\lambda_{2}$. Switching is inhibited outside the reflection profile of the grating because of symmetric cross-phase modulation from the counterpropagating control signals. The grating therefore confines nonlinear switching to only control-pulse wavelengths that lie inside its reflection band.

A schematic of the second experimental arrangement is shown in Fig. 3. In this case the NOLM was constructed with a 50/50 coupler and $11 \mathrm{~km}$ of dispersion-shifted fiber with a zero-dispersion wavelength $\lambda_{0}$ of $1551 \mathrm{~nm}$. Rejection of the control signal from one arm was achieved by use of a linear Bragg grating centered at $\lambda_{g}=1535 \mathrm{~nm}$ with a reflectivity of $95 \%$. Signal pulses at $1539 \mathrm{~nm}\left(\lambda_{2}\right)$ with a duration of 74 ps from a gain-switched distributedfeedback laser modulated at $76 \mathrm{MHz}$ were combined with 10-ps control pulses at $1535 \mathrm{~nm}\left(\lambda_{1}\right)$ from the color-center laser by use of a 90/10 coupler. The average power of the signal stream was $\sim 10 \mu \mathrm{W}$. The control-pulse walk-off length, i.e., the propagation distance required for the two signals to accumulate a relative delay of $10 \mathrm{ps}$, was $\sim 1.6 \mathrm{~km}$. We separated the residual control-pulse energy reflected by the grating from the switched signal stream by using a bulk diffraction grating and monitored the isolated signal by using a $22-\mathrm{GHz}$ photodiode.

The response of the device is summarized in Fig. 4. Trace (i) of Fig. 4(a) shows the temporal profile of a signal pulse emerging from the device, and trace (ii) shows the output in the presence of the control signal. It is clear that the introduction of the control pulses causes the output of the device to be significantly extinguished. The switching threshold was measured to be $2 \mathrm{~mW}$, which corresponds to a pulse energy of $26 \mathrm{pJ}$. This value agrees well with our predicted threshold that included fiber loss and was based on a simple cw cross-phase-modulation analysis for a strong control pulse on a weak signal. The measured extinction ratio at the transmission peak was $4.8 \mathrm{~dB}$. It was found that, by detuning the mode



Fig. 3. Schematic of the experimental arrangement to demonstrate bandpass two-wavelength switching. The distributed-feedback (DFB) laser pulse width is $74 \mathrm{ps}$. EDFA, erbium-doped fiber amplifier. 

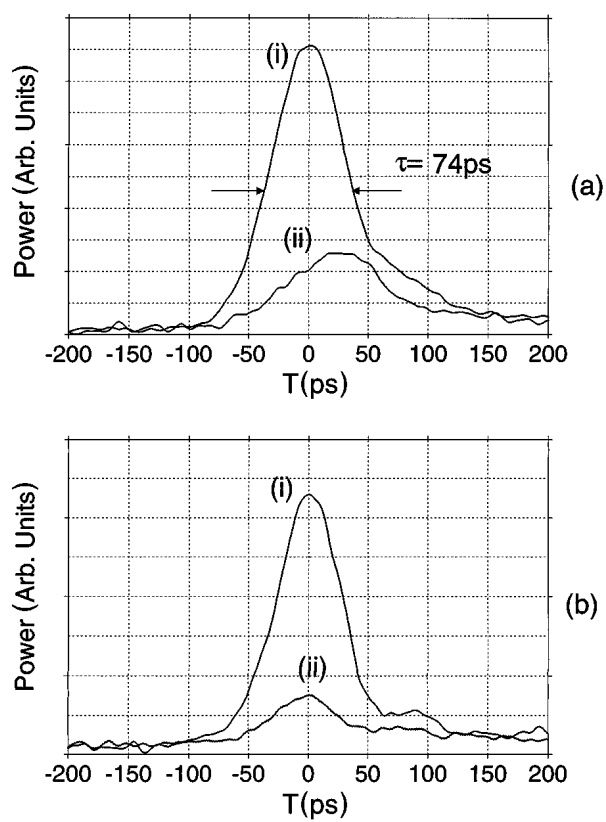

Fig. 4. (a) NOLM biased into linear transmission for outputs without [trace (i)] and with [trace (ii)] the control signal present. (b) NOLM biased into linear reflection for outputs with the control signal on [trace (i)] and off [trace (ii)] the grating.

locking of the color-center laser to produce longer control pulses, we could increase the extinction ratio to values greater than $7 \mathrm{~dB}$ as a result of the improved overlap between the signal and control pulses.

Trace (i) of Fig. 4(b) shows the nonlinearly switched portion of the input signal pulses. To demonstrate the bandpass nature of the nonlinear response, we tuned the wavelength of the control signal away from the grating peak to $1531 \mathrm{~nm}$. The resultant output is shown by trace (ii). The wavelength detuning required for extinguishing the response was $4 \mathrm{~nm}$, and the output shows an extinction ratio of $9.6 \mathrm{~dB}$. The small residual signal can be attributed to an imbalance in the coupler splitting ratio, since neither the control nor the signal wavelength corresponds precisely with the 50/50 point of the device $(\lambda=1550 \mathrm{~nm})$, producing a small differential nonlinearity in the arms. The results demonstrate that the nonlinear response of the device is confined to control-signal wavelengths inside the reflection band of the grating.

In conclusion, we have demonstrated a novel device configuration based on the combination of a NOLM and an in-fiber Bragg grating to specify the wave- length response of the NOLM. We have demonstrated self-switching in the soliton regime, using an in-fiber Bragg grating as a wavelength-dependent loss element, showing that the response of the device is limited to input signals determined by the grating reflection band. By increasing the reflectivity of the grating toward $100 \%$ we have confined a highpower control signal at the grating reflection peak to one arm of the device to demonstrate two-wavelength switching of a low-power signal stream. In this configuration the signal stream is switched only by a control signal coincident with the grating reflection band.

In both the self-switched and the control-pulseswitched schemes a single NOLM may be configured to operate on a set of specified wavelengths by the simple insertion of multiple gratings in the loop. In addition, the inclusion of a blazed (slantedfringe) fiber Bragg grating to couple light out of the fiber core would eliminate any possible resonance effects from the grating, thereby automatically separating the control and signal wavelengths. In this first demonstration we have achieved a wavelength discrimination of $4 \mathrm{~nm}$, which may be reduced by use of a more selective fiber grating. The use of gratings with sharp reflection band edges should result in smaller detuning values than those recorded here, further extending the potential of the device in high-density wavelength-division multiplexing systems and fast time-wavelength multiplexing.

\section{References}

1. N. Finlayson, B. K. Nayar, and N. J. Doran, Opt. Lett. 17, 112 (1992).

2. N. J. Doran and D. Wood, Opt. Lett. 13, 56 (1988).

3. M. E. Fermann, F. Haberl, M. Hofer, and H. Hochreiter, Opt. Lett. 15, 752 (1990).

4. K. J. Blow, N. J. Doran, B. K. Nayar, and B. P. Nelson, Opt. Lett. 15, 248 (1990).

5. D. M. Patrick, A. D. Ellis, and D. M. Spirit, Electron. Lett. 29, 702 (1993).

6. L. F. Mollenauer, E. Lichtman, M. J. Neubelt, and G. T. Harvey, Electron. Lett. 29, 910 (1993).

7. G. I. Stegeman, E. M. Wright, N. Finlayson, R. Zanoni, and C. T. Seaton, J. Lightwave Technol. 6, 953 (1988).

8. S. Larochelle, Y. Hibino, V. Mizrahi, and G. I. Stegeman, Electron. Lett. 26, 1459 (1990).

9. M. C. Farries, K. Sugden, D. C. J. Reid, I. Bennion, A. Molony, and M. J. Goodwin, Electron. Lett. 30, 891 (1994).

10. D. B. Mortimore, J. Lightwave Technol. 6, 1217 (1988). 